\title{
On the connections between multigranulation rough sets and generalized rough sets
}

\author{
Jiao Yang ${ }^{1,}$, Shiming Wan ${ }^{2}$, Keyun Qin ${ }^{3, b}$ \\ ${ }^{1}$ Chengdu Paotongshu Primary School (Tianfu Campus) Chengdu, 610094, China \\ ${ }^{2}$ School of Architecture and Civil Engineering, Chengdu University, Chengdu, 610106, China \\ ${ }^{3}$ College of Mathematics, Southwest Jiaotong University, Chengdu, 610106, China \\ aemail: 12303787@qq.com, bemail:keyunqin@263.net
}

Keywords: Rough Set; Generalized Rough Set; Pessimistic Multigranulation rough set; Optimistic Multigranulation Rough Set

\begin{abstract}
This note makes a comparative study of multigranulation rough set model and generalized rough set model. It is pointed out that the pessimistic multigranulation rough set model (PMGRS) is a kind of generalized rough set model, it is induced by a reflexive and symmetric relation on the universe. On the contrary, the optimistic multigranulation rough set model (OMGRS) is not a generalized rough set model. In other words, OMGRS can not be induced by a binary relation in general. Furthermore, some mistakes in the existing papers are pointed out.
\end{abstract}

\section{Pessimistic multigranulation rough sets}

Rough set theory, proposed by Pawlak [1], is a well-established mechanism for dealing with vagueness and uncertainty in data analysis. Rough set model is constructed on the basis of an approximation space $(U, R)$, where $U$ is a non-empty set of objects (also called the universe of discourse) and $R$ is an equivalence relation imposed upon $U$. An arbitrary subset of the universe (or target concept) is approximated by a pair of lower and upper approximations constructed by the equivalence classes associated with the equivalence relation $R$. The concepts of upper and lower approximations in rough set theory allow us to discover the knowledge hidden in information systems and express it in the form of decision rules.

In Pawlaks rough set model, equivalence relation is a key and primitive notion. The equivalence classes are building blocks for constructing the lower and upper approximations. This equivalence relation, however, seems to be a very stringent condition that may limit the application domain of the rough set model. To solve this problem, Pawlak's rough set model has been generalized to arbitrary binary relation based rough set model, covering based rough set model, fuzzy rough set model, etc, to meet the needs of some real application problems. Yao [2] proposed generalized rough set model and investigated the constructive and algebraic approaches in the study of rough sets. In the constructive approach, one starts from a binary relation on the universe and defines a pair of lower and upper approximation operators using the binary relation. In the algebraic approach, one defines a pair of dual approximation operators and states axioms that must be satisfied by the operators. The axioms of approximation operators guarantee the existence of certain types of binary relations producing the same operators.

Definition 1 [1] Let $U$ be a finite universe and $R$ an equivalence relation on $U$. The pair $(U, R)$ is referred to as a Pawlak approximation space. For any $X \subseteq U$, the lower approximation $\underline{R}(X)$ and the upper approximations $\bar{R}(X)$ of $X$ are defined by

$$
\begin{aligned}
& \underline{R}(X)=\left\{x \in U ;[x]_{R} \subseteq X\right\} \\
& \bar{R}(X)=\left\{x \in U ;[x]_{R} \cap X \neq \varnothing\right\}
\end{aligned}
$$

where $[x]_{R}=\{y \in U ;(x, y) \in R\}$ is the equivalence class with respect to $R$ which containing $x$. 
Definition 2 [2] Let $U$ be a finite universe and $R$ a binary relation on $U$. The pair $(U, R)$ is referred to as a generalized approximation space. For any $X \subseteq U$, the lower approximation $\underline{R}(X)$ and the upper approximations $\bar{R}(X)$ of $X$ are defined by

$$
\begin{aligned}
& \underline{R}(X)=\left\{x \in U ; R_{s}(x) \subseteq X\right\} \\
& \bar{R}(X)=\left\{x \in U ; R_{s}(x) \cap X \neq \varnothing\right\}
\end{aligned}
$$

where $R_{s}(x)=\{y \in U ;(x, y) \in R\}$ is referred to as the successor neighborhood of $x$ with respect to $R$.

In this definition, for any $X \subseteq U$, we have

$$
\sim \bar{R}(\sim X)=\sim\left\{x \in U ; R_{s}(x) \cap(\sim X) \neq \varnothing\right\} \quad=\left\{x \in U ; R_{s}(x) \cap X \neq \varnothing\right\}=\bar{R}(X)
$$

where $\sim X=U-X$ is the complement of $X$. Thus, $\underline{R}$ and $\bar{R}$ are dual to each other. Additionally, if $R$ is an equivalence relation, then $R_{s}(x)=[x]_{R}$. Thus, Definition 2 is a generalization of Definition 1.

Theorem 1 [2] Suppose $U$ is a non-empty set and $L, H: P(U) \rightarrow P(U)$ are dual unary operators, i.e., $L(X)=\sim H(\sim X)$ for each $X \subseteq U$. There exists a binary relation $R$ on $U$ such that $L(X)=\underline{R}(X)$ and $H(X)=\bar{R}(X)$ for all $X \subseteq U$, if and only if $L$ and $H$ satisfy the properties:

$(\mathrm{L} 1): L(U)=U$,

(L2): $L(X \cap Y)=L(X) \cap L(Y)$;

(H1) : $H(\varnothing)=\varnothing$,

$(\mathrm{H} 2): H(X \cup Y)=H(X) \cup H(Y)$.

Additionally, there exists a transitive relation $R$ on $U$ such that $L(X)=\underline{R}(X)$ and $H(X)=\bar{R}(X)$ for all $X \subseteq U$, if and only if $L$ and $H$ satisfy (L1),(L2),(H1),(H2) and

(L3) : $L(X) \subseteq L(L(X))$,

(H3) : $H(H(X)) \subseteq H(X)$.

In this Theorem, $L$ and $H$ are referred to as approximation operators induced by $R$.

Knowledge representation in the rough set model is realized via information systems. An information system is an ordered triple $S=(U, A T, f)$, where $U$ is a finite non-empty set of objects, $A T$ is a finite non-empty set of attributes, $f=\left\{f_{a} ; a \in A T\right\}$ and $f_{a}: U \rightarrow V_{a}$ for any $a \in A T$ with $V_{a}$ being the domain of an attribute $a$. For any $P \subseteq A T, P$ determines an indiscernibility relation $\hat{P}$ on $U$ as follows:

$$
\hat{P}=\left\{(x, y) \in U^{2} ; \forall a \in P\left(f_{a}(x)=f_{a}(y)\right)\right\}
$$

Clearly, $\hat{P}$ is an equivalence relation. We denote by $[x]_{P}$ the equivalence class containing $x$.

In view of granular computing, an equivalence relation on the universe can be regarded as a granulation. The set of related equivalence classes forms a granulation space. It can thus be concluded that Pawlak's rough set model is based on a single granulation [3]. Qian and Liang extended Pawlak's single granulation rough set model to a multigranulation rough set by using multi-equivalence relations. The optimistic multigranulation rough set model and the pessimistic multigranulation rough set model are presented $[4,5]$. Recently, more attentions have been paid to multigranulation rough sets $[6,7,8,9,10]$.

Definition 3 [4] Let $S=(U, A T, f)$ be an information system, $A_{1}, \cdots, A_{m} \subseteq A T, X \subseteq U$. The pessimistic lower approximation ${\underline{\sum_{i=1}^{m}}}_{i}^{p}(X)$ and the pessimistic upper approximation $\overline{\sum_{i=1}^{m} A_{i}^{p}}(X)$ of $X$ with respect to $A_{1}, \cdots, A_{m}$ are defined by

$$
\begin{aligned}
& \underline{\underline{\sum_{i=1}^{m} A_{i}^{p}}}(X)=\left\{x \in U ;[x]_{A_{1}} \subseteq X \wedge \cdots \wedge[X]_{A_{m}} \subseteq X\right\} \\
& \overline{\sum_{i=1}^{m} A_{i}^{p}}(X)=\sim \underline{\sum_{i=1}^{m} A_{i}^{p}(\sim X)}
\end{aligned}
$$

The ordered pair $\left(\underline{\sum_{i=1}^{m} A_{i}^{p}}(X), \overline{\sum_{i=1}^{m} A_{i}^{p}}(X)\right)$ is called the pessimistic multigranulation rough sets of 
$X$. Here, the word pessimistic means that all granular structures are needed to satisfy with the inclusion condition between an equivalence class and a target concept when multiple granular structures are available.

We have the following properties of pessimistic multigranulation rough sets:

(1) $\underline{\sum_{i=1}^{m} A_{i}^{p}}(U)=U=\overline{\sum_{i=1}^{m} A_{i}^{p}}(U)$.

(2) $\underline{\sum_{i=1}^{m} A_{i}^{p}}(\varnothing)=\varnothing=\overline{\sum_{i=1}^{m} A_{i}^{p}}(\varnothing)$.

(3) $\underline{\sum_{i=1}^{m} A_{i}^{p}}(X) \subseteq X \subseteq \overline{\sum_{i=1}^{m} A_{i}^{p}}(X)$.

(4) $X \subseteq Y$ implies that $\underline{\sum_{i=1}^{m} A_{i}^{p}}(X) \subseteq \underline{\sum_{i=1}^{m} A_{i}^{p}}(Y)$ and $\overline{\sum_{i=1}^{m} A_{i}^{p}}(X) \subseteq \overline{\sum_{i=1}^{m} A_{i}^{p}}(Y)$.

(5) $\underline{\sum_{i=1}^{m} A_{i}^{p}}(X)=\cap_{i=1}^{m} \underline{\hat{A}_{i}}(X) \overline{\sum_{i=1}^{m} A_{i}^{p}}(X)=\cup_{i=1}^{m} \overline{\hat{A}_{i}}(X)$.

(6) $\underline{\sum_{i=1}^{m} A_{i}^{p}}\left(\cap_{j=1}^{n} X_{j}\right)=\cap_{j=1}^{n} \underline{\sum_{i=1}^{m} A_{i}^{p}}\left(X_{j}\right), \overline{\sum_{i=1}^{m} A_{i}^{p}}\left(\cup_{j=1}^{n} X_{j}\right)=\cup_{j=1}^{n} \overline{\sum_{i=1}^{m} A_{i}^{p}}\left(X_{j}\right)$.

We consider the connections between pessimistic multigranulation rough sets and generalized rough sets. Let $S=(U, A T, f)$ be an information system, $A_{1}, \cdots, A_{m} \subseteq A T$. We denote $A=\left\{A_{1}, \cdots, A_{m}\right\}$ and define binary relation $R_{A}$ on $U$ as follows:

$$
R_{A}=\left\{(x, y) ; \exists i\left((x, y) \in \widehat{A}_{i}\right)\right\}
$$

i.e., $(x, y) \in R_{A}$ if and only if there exists $i$ such that $f_{a}(x)=f_{a}(y)$ for each $a \in A_{i}$. Clearly, $R_{A}$ is a reflexive and symmetric relation on $U$.

Theorem 2 Let $S=(U, A T, f)$ be an information system, $A_{1}, \cdots, A_{m} \subseteq A T, A=\left\{A_{1}, \cdots, A_{m}\right\}$. For any $X \subseteq U$,

(1) $\underline{\sum_{i=1}^{m} A_{i}^{p}}(X)=\underline{R_{A}}(X)$.

(2) $\overline{\sum_{i=1}^{m} A_{i}^{p}}(X)=\overline{R_{A}}(X)$.

Proof. (1) For each $x \in U$, we have $R_{A_{s}}(x)=[x]_{A_{1}} \cup \cdots \cup[x]_{A_{m}}$. In fact, for any $y \in R_{A_{s}}(x)$, it follows that $(x, y) \in R_{A}$ and thus there exists $i$ such that $(x, y) \in \widehat{A}_{i}$. Therefore, $y \in[x]_{A_{i}} \subseteq[x]_{A_{1}} \cup \cdots \cup[x]_{A_{m}}$. Conversely, if $y \in[x]_{A_{1}} \cup \cdots \cup[x]_{A_{m}}$, then there exists $i$ such that $y \in[x]_{A_{i}}$ and hence $(x, y) \in \widehat{A}_{i}$, i.e., $(x, y) \in R_{A}$ and $y \in R_{A_{s}}(x)$.

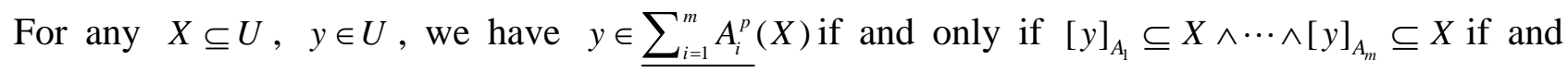
only if $[y]_{A_{1}} \cup \cdots \cup[y]_{A_{m}} \subseteq X$ if and only if $R_{A_{s}}(x) \subseteq X$ if and only if $y \in \underline{R_{A}}(X)$. Thus $\underline{\sum_{i=1}^{m} A_{i}^{p}}(X)=\underline{R_{A}}(X)$ as required.

(2)For any $X \subseteq U$, by the duality of lower and upper approximation operators, we have

$$
\overline{\sum_{i=1}^{m} A_{i}^{p}}(X)=\sim \underline{\sum_{i=1}^{m} A_{i}^{p}}(\sim X)=\sim \underline{R_{A}}(\sim X)=\overline{R_{A}}(X) .
$$

This theorem shows that pessimistic multigranulation rough set model is a kind of generalized 
rough set model in the sense of Definition 1. It is induced by the binary relation $R_{A}$.

Example 1 Suppose that $S=(U, A T, f)$ is an information system, where $U=\left\{e_{1}, e_{2}, \cdots, e_{8}\right\}, L, P \subseteq A T$ determine indiscernibility relations $\hat{L}, \hat{P}$ on $U$. The partitions induced by $\hat{L}$ and $\hat{P}$ are as follows $\hat{L}=\left\{\left\{e_{1}, e_{7}\right\},\left\{e_{2}, e_{3}, e_{4}, e_{5}, e_{6}\right\},\left\{e_{8}\right\}\right\} \quad \hat{P}=\left\{\left\{e_{1}, e_{2}\right\},\left\{e_{3}, e_{4}, e_{5}\right\},\left\{e_{6}, e_{7}, e_{8}\right\}\right\}$. Let $A=\{L, P\} . A$ induces binary relation $R_{A}$. According to (6), the successor neighborhood of $x \in U$ with respect to $R_{A}$ are as follows:

$$
\begin{aligned}
& R_{\mathrm{A}_{5}}\left(e_{1}\right)=\left\{e_{1}, e_{2}, e_{7}\right\}, R_{A_{5}}\left(e_{2}\right)=\left\{e_{1}, e_{2}, e_{3}, e_{4}, e_{5}, e_{6}\right\}, R_{A_{5}}\left(e_{3}\right)=R_{A_{5}}\left(e_{4}\right)=R_{A_{5}}\left(e_{5}\right)=\left\{e_{2}, e_{3}, e_{4}, e_{5}, e_{6}\right\}, \\
& R_{A_{5}}\left(e_{6}\right)=\left\{e_{2}, e_{3}, e_{4}, e_{5}, e_{6}, e_{7}, e_{8}\right\}, R_{A_{5}}\left(e_{7}\right)=\left\{e_{1}, e_{6}, e_{7}, e_{8}\right\}, R_{A_{5}}\left(e_{8}\right)=\left\{e_{6}, e_{7}, e_{8}\right\} .
\end{aligned}
$$

Let $X=\left\{e_{6}, e_{7}, e_{8}\right\}$. By direct computation, we have

$$
\underline{\sum_{i=1}^{m} A_{i}^{p}}(X)=\underline{R_{A}}(X)=\left\{e_{8}\right\}, \quad \underline{\sum_{i=1}^{m} A_{i}^{p}}\left(\underline{\sum_{i=1}^{m} A_{i}^{p}}(X)\right)=\underline{R_{A}}\left(\underline{R_{A}}(X)\right)=\varnothing .
$$

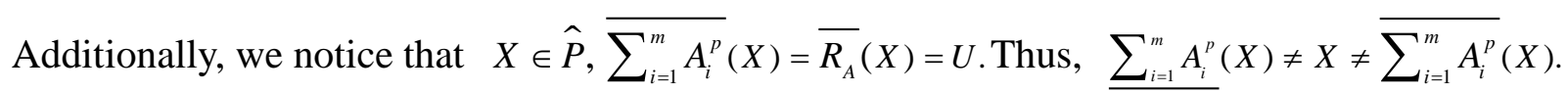

This example shows that, in pessimistic multigranulation rough set model, $\underline{\sum_{i=1}^{m} A_{i}^{p}}(X)=\underline{\sum_{i=1}^{m} A_{i}^{p}}\left(\underline{\sum_{i=1}^{m} A_{i}^{p}}(X)\right)$ and $\overline{\sum_{i=1}^{m} A_{i}^{p}}\left(\sum_{i=1}^{m} A_{i}^{p}(X)\right)=\overline{\sum_{i=1}^{m} A_{i}^{p}}(X)$ do not hold in general. Thus, (6PMH), (9PML) and (9PMH) in [6] (Page 105) and Proposition 2(3) in [9] (Page 83) are not correct.

In this example, $\left(e_{1}, e_{2}\right) \in R_{A},\left(e_{2}, e_{3}\right) \in R_{A}$ and $\left(e_{1}, e_{3}\right) \notin R_{A}$. It shows that $R_{A}$ is not transitive in general. Therefore, it is also concluded that, by the axiomatic approach presented in [2] (Theorem 6, Page 31), the idempotency of pessimistic multigranulation rough approximation operators does not hold in general.

\section{Optimistic multigranulation rough sets}

In this section, we consider optimistic multigranulation rough set model.

Definition 4 [5] Let $S=(U, A T, f)$ be an information system, $A_{1}, \cdots, A_{m} \subseteq A T, X \subseteq U$. The

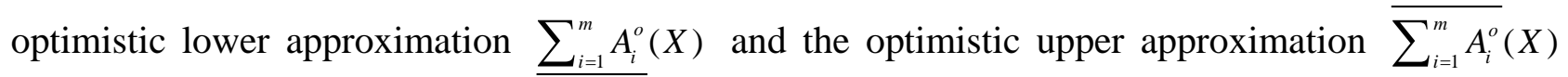
of $X$ with respect to $A_{1}, \cdots, A_{m}$ are defined by

$$
\begin{aligned}
& \underline{\underline{\sum_{i=1}^{m} A_{i}^{o}}}(X)=\left\{x \in U ;[x]_{A_{1}} \subseteq X \vee \cdots \vee[X]_{A_{m}} \subseteq X\right\} \\
& \overline{\sum_{i=1}^{m} A_{i}^{o}}(X)=\sim \underline{\underline{\sum_{i=1}^{m} A_{i}^{o}}(\sim X)}
\end{aligned}
$$

The ordered pair $\left(\underline{\sum_{i=1}^{m} A_{i}^{o}}(X), \overline{\sum_{i=1}^{m} A_{i}^{o}}(X)\right)$ is referred to as the optimistic MGRS. Here, the word optimistic means that only one granular structure is needed to satisfy with the inclusion condition between an equivalence class and a target concept when multiple granular structures are available in problem processing.

Let $S=(U, A T, f)$ be an information system, $A_{1}, \cdots, A_{m} \subseteq A T$. For any $X, Y \subseteq U$, we have the following properties of pessimistic multigranulation rough sets [5]:

(1) $\underline{\sum_{i=1}^{m} A_{i}^{o}}(U)=U=\overline{\sum_{i=1}^{m} A_{i}^{o}}(U)$. 
(2) $\underline{\sum_{i=1}^{m} A_{i}^{o}}(\varnothing)=\varnothing=\overline{\sum_{i=1}^{m} A_{i}^{o}}(\varnothing)$.

(3) $\sum_{i=1}^{m} A_{i}^{o}(X) \subseteq X \subseteq \overline{\sum_{i=1}^{m} A_{i}^{o}}(X)$.

(4) $X \subseteq Y$ implies that $\underline{\sum_{i=1}^{m} A_{i}^{o}}(X) \subseteq \underline{\sum_{i=1}^{m} A_{i}^{o}}(Y)$ and $\overline{\sum_{i=1}^{m} A_{i}^{o}}(X) \subseteq \overline{\sum_{i=1}^{m} A_{i}^{o}}(Y)$.

(5) $\underline{\sum_{i=1}^{m} A_{i}^{o}}(X)=\cup_{i=1}^{m} \underline{\hat{A}_{i}}(X) \overline{\sum_{i=1}^{m} A_{i}^{o}}(X)=\cap_{i=1}^{m} \overline{\hat{A}_{i}}(X)$.

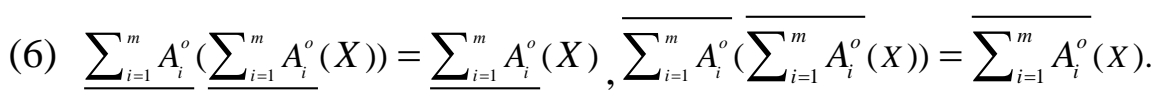

Example 2 We consider the information system $S=(U, A T, f)$ given in Example 1. Let $X=\left\{e_{1}, e_{7}, e_{8}\right\}, Y=\left\{e_{6}, e_{7}, e_{8}\right\}$. By direct computation, we have

$$
\begin{aligned}
& \underline{\sum_{i=1}^{m} A_{i}^{o}}(X)=\left\{e_{1}, e_{7}, e_{8}\right\}, \underline{\sum_{i=1}^{m} A_{i}^{o}}(Y)=\left\{e_{6}, e_{7}, e_{8}\right\}, \\
& \underline{\sum_{i=1}^{m} A_{i}^{o}}(X \cap Y)=\underline{\sum_{i=1}^{m} A_{i}^{o}}\left(\left\{e_{7}, e_{8}\right\}\right)=\left\{e_{8}\right\} .
\end{aligned}
$$

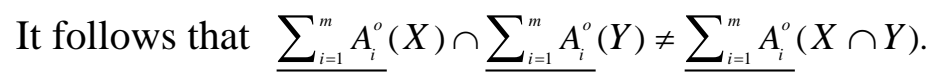

This example shows that the optimistic lower approximation operator $\sum_{i=1}^{m} A_{i}^{o}$ does not preserve set intersection in general. By Theorem 1, there may not necessarily exist a binary relation $R$ on $U$ such that $\underline{\sum_{i=1}^{m} A_{i}^{o}}(X)=\underline{R}(X)$ and $\overline{\sum_{i=1}^{m} A_{i}^{o}}(X)=\bar{R}(X)$ for each $X \subseteq U$. In other words, the optimistic multigranulation approximation operators can not be induced by a binary relation in general.

Zhang [10] proposed an algebraic approach for the rough approximation operators in the multiple approximation spaces. Four kinds of constructive methods of rough approximation operators from existing rough sets are established, and some important properties are investigated.

Let $U$ be a nonempty set, $\underline{a p r}{ }^{(i)}$ and $\overline{a p r}^{(i)}$ some mappings from $P(U)$ to $P(U), i=1, \cdots, n$. Four pairs of new mappings from $P(U)$ to $P(U)$ as defined follows: for any $X \subseteq U$,

(1) $(\cup, \cap)$-type operators:

$$
\underline{\operatorname{apr}}_{(\cup, \cap)}^{1 \leq i \leq n}(X)=\cup_{i=1}^{n} \underline{a p r}^{(i)}(X), \quad \overline{\operatorname{apr}}_{(\cup, \cap)}^{1 \leq i \leq n}(X)=\cap_{i=1}^{n} \overline{\operatorname{apr}}^{(i)}(X) .
$$

(2) $(\cap, \cup)$-type operators:

$$
\underline{\operatorname{apr}}_{(\cap, \cup)}^{1 \leq i \leq n}(X)=\cap_{i=1}^{n} \underline{\operatorname{apr}}^{(i)}(X), \overline{\operatorname{apr}}_{(\cap, \cup)}^{1 \leq i \leq n}(X)=\cup_{i=1}^{n} \overline{\operatorname{apr}}^{(i)}(X) .
$$

(3) $(\cap, \cap)$-type operators:

$$
\underline{\operatorname{apr}}_{(\cap, \cap)}^{1 \leq i \leq n}(X)=\cap_{i=1}^{n} \underline{\operatorname{apr}}^{(i)}(X), \overline{\operatorname{apr}}_{(\cap, \cap)}^{1 \leq i \leq n}(X)=\cap_{i=1}^{n} \overline{\operatorname{apr}}^{(i)}(X) .
$$

(4) $(\cup, \cup)$-type operators:

$$
\underline{\operatorname{apr}}_{(\cup, \cup)}^{1 \leq i \leq n}(X)=\cup_{i=1}^{n} \underline{\operatorname{apr}}^{(i)}(X), \overline{\operatorname{apr}}_{(\cup, \cup i \leq n}^{1 \leq n}(X)=\cup_{i=1}^{n} \overline{\operatorname{apr}}^{(i)}(X) .
$$

Let $S=(U, A T, f)$ be an information system, $A_{1}, \cdots, A_{m} \subseteq A T$. It is noticed that [10], if $\underline{\operatorname{apr}^{(i)}}$ and $\overline{\operatorname{apr}}^{(i)}$ are the lower and upper approximation operators induced by equivalence relation $\widehat{A}_{i}$, 
that is $\underline{a p r}^{(i)}(X)=\underline{\widehat{A}_{i}}(X)$ and $\overline{\operatorname{apr}}(i)(X)=\overline{\widehat{A}_{i}}(X)$ for each $X \subseteq U$. Then we conclude that $(\cap$, $\cup$ )-type operators is a kind of pessimistic multigranulation rough approximation operator. Thus, it can be induced by a reflexive and symmetric binary relation on the universe. In other words, it is a kind of generalized approximation operators. On the other hand, $(\cup, \cap)$-type operator is a kind of optimistic multigranulation rough approximation operator. It follows that $(\cup, \cap$ )-type operator can not be induced by a binary relation on $U$. As for $(\cap, \cap)$-type operators and $(U, U)$-type operators, we noticed that they are not dual to each other. Thus, they are not generalized rough approximation operators in the sense of Definition 2 .

\section{Conclusion}

This note makes a comparative study of multigranulation rough set and generalized rough set induced by binary relations. It is pointed out that the pessimistic multigranulation rough set model (PMGRS) is a kind of generalized rough set model induced by a reflexive and symmetric relation. On the contrary, the optimistic multigranulation rough set model (OMGRS) is not a generalized rough set model. It can not be induced by a binary relation in general. Furthermore, some mistakes in the existing papers are pointed out.

\section{Acknowledgement}

This work has been partially supported by the National Natural Science Foundation of China (Grant No. 61473239).

\section{References}

[1] Z.Pawlak, Rough sets, International Journal of Computer and Information Sciences, 11(1982) 341-356.

[2] Y.Y.Yao, Constructive and algebraic methods of the theory of rough sets, Journal of Information Sciences, 109(1998)21-47.

[3] Y.H.Qian, J.Y.Liang, Rough set method based on multi-granulations, in: Proc. 5th IEEE International Conference on Cognitive Informatics, Beijing, China, July 17-19, 2006, pp.297-304.

[4] Y.H.Qian, J.Y.Liang, W.Wei, Pessimistic rough decision, Journal of Zhejiang Ocean University, 5(2010) 440-449.

[5] Y.H.Qian, J.Y.Liang, Y.Y.Yao, C.Y.Dang, MGRS: A multi-granulation rough sets, Information Sciences, 180(2010)949-970.

[6] G.P.Lin, J.Y.Liang, Y.H.Qian, Multigranulation rough sets: From partition to covering, Information Sciences, 241(2013)101-118.

[7] C.H.Liu, D.Q.Miao, J.Qian, On multi-granulation covering rough sets, International Journal of Approximate Reasoning, J.Y.Liang, Y.H.Qian, Multigranulation rough sets: From partition to covering, Information Sciences, 55(2014)1404-1418.

[8] C.H.Liu, D.Q.Miao, J.Qian, On multi-granulation covering rough sets, International Journal Cybern., Part A 20(2010)420-430.

[9] Y.H.She, X.L.He, On the structure of the multigranulation rough set model, Knowledge-Based Systems, 36(2012)81-92.

[10] X.H.Zhang, D.Q.Miao, M.L.Le, Constructive methods of rough approximation operators and multigranulation rough sets, Knowledge-Based Systems, 91(2016)114-125. 